\title{
Research Findings and Strategies for Assessing Telemedicine Costs
}

\author{
TIM REARDON, Ph.D.
}

\begin{abstract}
With varying degrees of enthusiasm, researchers and decision-makers support the use of telemedicine. Forms of telemedicine are appearing in health-care delivery, and are often integral to transforming health-care information technology. Despite this, the appropriate role of telemedicine in the delivery process remains ambiguous, at least partly because of its uncertain impact on costs. Cost savings and benefits are often suggested by the logic of its impact on health care and by the promise of technology, but definitive information on the costs and benefits remain elusive. The objectives of this paper are to review the state of telemedicine cost research, to examine major issues affecting the yield from this research, and finally to recommend strategies for improving future research. As this paper demonstrates, the productivity of telemedicine cost studies suffers from an under-utilization of appropriate program evaluation and economic methods. This review of telemedicine cost literature will appraise telemedicine cost studies and their findings within a broad analytic framework. Telemedicine cost studies will be assessed on their methods of statistical inference, use of critical economic concepts, and contextual definition for the determination of costs and benefits.
\end{abstract}

\section{OBJECTIVES AND BACKGROUND}

$\mathbf{R}$ ESEARCHERS HAVE YET TO ESTABLISH either a sufficient body of findings or a vision for understanding telemedicine costs. Systematic literature reviews report a few useful cost findings along with many inadequate methods of inference. Most notable is the absence of randomized, controlled trials. But even among studies meeting minimal methodological criteria, studies suffer from poor use of analytic techniques in economics and inadequate documentation.

Difficulties in cost analysis have been attributed to problems in evaluating new programs, low levels of use, and rapidly changing technology. A few authors have called into question the usefulness of traditional tools of economic evaluation and have proposed new conceptual frameworks. ${ }^{1}$ Despite these appraisals, there are no detailed assessments or proposed strategies for addressing the low productivity of telemedicine cost analysis, whether from inadequate use of theory, the difficult nature of applying economics in this field, or limited expertise in the requisite methodology.

Clinical research design and statistical inference have guided telemedicine cost evaluations. The methodological benchmark has compared assessments of alternatives, and the 
strongest evidence and highest-quality findings come from randomized, controlled trial (RCT) designs. Systematic reviews of telemedicine literature largely are based on criteria from classic RCT, and comparisons are among alternative interventions as measured by costs and benefits. But although the RCT model is appropriate for assessing clinical effectiveness, the evaluation of telemedicine programs has fundamentally different purposes, standards for judging causality, logistical constraints, and implications from major threats to validity. ${ }^{2}$ Hence different methods may enable more effective program cost evaluations.

RCT guarantees little when applied to most telemedicine program evaluation, which faces different constraints from clinical research. Research on pharmaceutical and biological devices, for example, depends on the use of RCT methods to infer causality. On the other hand, the purpose of telemedicine cost evaluation is to support program and policy decision making.

The overall quality and success of a program evaluation depends on understanding and applying findings to decision-making contexts and needs. The key methodological challenge in telemedicine cost evaluation is to identify the decision-making information requirements and to depict the decision framework that defines the contextual validity of findings. This issue was argued in a recent position paper of the American Evaluation Association (AEA),

RCTs are not always best for determining causality and can be misleading. RCTs examine a limited number of isolated factors that are neither limited nor isolated in natural settings. The complex nature of causality and the multitude of actual influences on outcomes render RCTs less capable of discovering causality than designs sensitive to local culture and conditions and open to unanticipated causal factors. $^{2}$

Consequently, although selecting a methodology that achieves internal validity remains important in cost evaluations, methods that fit the specific problem context and decision-making needs are equally important.
In addition, the effective interpretation and generalization of program cost evaluations rely on different tools and applications of conceptual schema as compared with those of clinical research and most cost evaluations of drugs and devices. Telemedicine programs are highly heterogeneous interventions, with complex sets of input, output, and context factors. Interpreting the data and findings on telemedicine program costs requires insight into the health-care delivery processes that generate the data. Interpretation, integration of findings, and generalizations often require insights from health-care delivery and economic theories. Meaning and differences in findings may not become apparent until viewed within a construct, such as health production.

At the same time, the specific uses of program cost evaluations shape the results and the applicability of findings. For example, cost evaluation may focus on providing results for a health-care organization or a system of care, or they may be intended for general application. Different research methods may provide equally valid information on a question, but the measures and control of contextual factors may require different evaluation methods. A review of research studies needs to incorporate the intended use of the evaluation. Different strategies may be needed to synthesize disparate findings.

Accordingly, telemedicine cost evaluations and reviews of this literature have not paid attention to the unique purposes, needs, and methods of program cost evaluation, nor have they focused on non-RCT or economic methodologies. Program cost evaluations involve matching analytic methods to the purpose and constraints of the evaluation, identifying the role and characteristics of decision-making frameworks, and using appropriate economic concepts for interpreting program findings. Each of these components of the analytic structure may be momentous in determining the value of a study. Although trade-offs exist in the use of optimal strategies, rigid adherence to strong methods of determining causality may make studies meaningless because of associated limitations in contextual validity. The productivity of telemedicine cost studies suffer from an under-utilization of appropriate pro- 
gram evaluation and economic methods in their design and assessment. First, however, these elements of cost evaluation are applied in the literature, and the methods of selecting the literature are described.

\section{ELEMENTS OF COST EVALUATIONS OF HEALTH-CARE SERVICES}

A review of the major analytic components of health-care services cost evaluations provides an overview of the economic concepts, cost analysis decision framework, and methods of inference that affect the quality and productivity of telemedicine cost research. This overview guides the subsequent identification, selection, and review of the telemedicine cost literature.

Cost evaluations of health-care services differ in their purpose. Information about costs may be needed in organizational resource planning; funding agencies may require evaluations to justify expenditures; or health-care system policy makers may be considering broad deployment of a technology. But at the core of each cost evaluation is the analytic contribution of economics, a comparison of alternatives based on the concept of "opportunity costs," defined in the MIT Dictionary of Modern Economics as "the value of resources given up through a decision." 56 The essence of the cost evaluation is identifying the alternative that provides the greatest value in relation to the opportunity costs. This may be described in three basic steps:

1. Identify and aggregate the costs of each health-care alternative.

2. Identify and aggregate valued outcomes of each alternative.

3. Compare the aggregated relative, "value for cost," for each alternative (e.g., such as the ratio of costs to changes in quality-adjusted life years attributable to an intervention).

This process seems simple; however, most of the debate around telemedicine cost analysis comes from difficulties in implementation, and here economic theory only provides broad guidance. The usual errors and shortcomings in the telemedicine cost literature may be attributable to its deceptive conceptual simplicity.

Implementation of a cost analysis requires a well-defined decision framework that consists of objectives, resources used, definitions of outputs, and a context of the decision making, such as timeframe. The relative significance of each these issues in determining the quality of a study depends on program characteristics and types of telemedicine.

Although the specification of telemedicine is grounded in economic concepts, it often depends on a broad, multidisciplinary understanding of health services delivery. Complex interventions of telemedicine, concepts of systems analysis, organizational decision making and strategy, and health-care behavior may be critical for identifying and understanding the value and cost of the telemedicine.

Differences of opinion exist regarding the value of applying economic concepts to cost evaluation. Some variations in methods may arise because of differences in objectives, assessments of the literature, or simply due to multiple, equally acceptable approaches on some issues. On the other hand, conformity in an approach facilitates assessments of literature and provides common utility from a cost study, especially if the study fits within broader research or health-care policy objectives. Consensus definitions and methods in several areas leverage the cost literature and provide collective value. Consensus guidelines are making their way into recommended cost evaluations, and they provide an import element in assessing telemedicine costs.

The following sections provide an overview of the cost evaluation that affects the quality of the telemedicine cost literature. Economic conceptual issues, the decision framework, related considerations of methodological inference, and the role of cost guidelines as they affect the quality of telemedicine cost studies are summarized. These materials will shape the subsequent review of the telemedicine literature.

\section{Economic concepts}

Economic theory, as applied to cost evaluation, generally is not a basis for prediction, but 
it provides concepts and a structure for identifying and developing those measures of inputs and outputs that define program performance. Primarily, the concepts of economic theory of choice constitute a reference for researchers developing observable measures of costs and benefits, and selecting the most appropriate cost evaluation model, such as cost-benefit analysis (CBA) and cost effectiveness analysis (CEA). Otherwise, for program cost evaluations, economic theories of organization and production may help clarify appropriate definitions; identify appropriate aggregations of costs and benefits, and interpret expected impact or findings.

\section{Economic costs and benefits}

The most important role of economic theory in cost evaluation is in guiding the identification and measurement of economic costs and benefits. In particular, the concept of costs, defined as an opportunity cost in the economic theory of choice, provides the conceptual foundation for defining costs as the value placed on those resources given up by a decision maker when making a specific choice. As such, costs identified in an economic evaluation are subjective, being defined distinctly from the perspective of a single decision maker.

However, costs, or components of costs, may be estimated through such metrics as market prices. A parallel concept may be applied to the valued gains of a decision that define benefits. Thus, among choice alternatives, a comparison is made of the estimates of a decision maker's opportunity costs relative to the value of outcomes. The measurement of costs is the negative aspect of choice, or the value of what is given up, and the measurement of benefits is the positive value placed on gains. The assumed preference is for the greatest value of outcomes relative to opportunity costs for the decision maker.

Of course, whereas the concept is deceptively reasonable and easy to grasp, the implementation is exasperatingly complex. To begin, the analyst must carefully define the decision maker and the intervention or decision under consideration and its alternatives. Economic concepts may help define relevant features of a telemedicine intervention, but mostly the de- scription and detail of a program are distinct from the core application of economic concepts in the cost evaluation. These issues of program definition are addressed in a subsequent section of this review. This task aside, however, the challenge lies in distinguishing inputs and outputs and assigning appropriate costs and benefits where they are tied to the subjective assessments of the decision maker.

The measurement of costs begins with an identification of the resources "given up" as a result of a choice, by implementing a telemedicine activity or program. Here, the question that needs careful consideration is whether implementation of the telemedicine program requires new resources or expenditures (e.g., construction) or the commitment of resources (e.g., existing facilities) that could be used for other purposes elsewhere. The concept of opportunity cost distinguishes the relevant cost for the evaluation in that the resources used are a consequence of the decision. For example, the institutional overhead of a program may be an accountant's cost, but not necessarily an economic cost because additional commitment of overhead may not be required with the implementation of telemedicine. Of course, this assessment of costs depends on the timeframe of the decision. In the short run, there may not be an opportunity cost for the facility, but longrun facility costs may be incurred. Hence, this assessment of program costs must consider the relevant timeframe.

Once opportunity costs are identified for a telemedicine program, the analyst is then able to address the related question on the value of the resources. Again, the value assigned to resources is defined from the perspective of the decision maker. Values may be easily assigned to some resources, as market prices often reasonably represent the value of resources such as equipment, manpower, etc. On the other hand, resource costs of volunteer labor and patient travel may not be easily determined. Nonetheless, an analytic objective should identify all resource costs (market and nonmarket) that accrue to a decision maker as a result of the program. A common metric of value must be used to produce an aggregate measure of costs for each program alternative. 
At the same time, each program choice, such as telemedicine or its alternative, results in outcomes as well as costs. Hence, the analysis must identify all of the important outcomes or gains from each intervention.

Identifying and then measuring the value or benefits of a telemedicine intervention may be considered the complement of determining the opportunity costs of resources, but this task in the evaluation assessment is often more challenging. In contrast to input costs, market prices are usually not available for the benefits derived from the health-care program. Moreover, the outputs themselves, such as a successful treatment or improvements in health frequently are not easily defined, identified, or measured.

Telemedicine programs may create new outcomes of care or change outputs or outcomes. These benefits of telemedicine are often difficult to identify, partly because detecting them depends on our expectations or knowledge of effects on health or other outcomes. Also, detecting benefits may depend on timing, with some benefits only becoming apparent at a time in the future. Then, again, whether an outcome is a benefit may depend on the perspective of the decision maker or stakeholder. The decision maker may be interested in the ability of telemedicine to provide visits or treat episodes of care, but not in the longer-term effects on health.

But even with outcomes identified, a formidable task remains in assigning value to the outcomes of telemedicine and aggregating the sum of benefits for all outcomes. From the perspective of economic analysis the ideal is to evaluate all outcomes with a common monetary metric. These could then be aggregated and compared with costs for each alternative. This type of assessment, CBA, is the preferred method of evaluating alternatives because it facilitates the most direct and ideal implementation of the economic evaluation paradigm, and the broadest consideration of cost and benefit tradeoffs, allowing comparisons with all similarly evaluated choice alternatives. Yet, the application of CBA is often difficult, especially in health care, because of the need to identify, measure, summarize, and compare outcomes that are not usually assessed through the mar- ket-based exchange. In particular, there is a general reluctance to assign a value to life or health, at least in such a way as to make it comparable to values placed on non-health-care commodities. Consequently, CBA that do not rely on monetary assessments of benefits are more often used in health-care cost evaluation.

Several alternative methods of CBA have evolved. Most are prominently distinguished by their methods of establishing and aggregating benefits, or valued outcomes. Each may be appropriate, depending on the needs of the analyst and the trade-offs. More than 10 government and industry organizations have developed health-care cost-analysis guidelines in which several different types of cost evaluations are recommended. ${ }^{3}$ Issues associated with several of the most common cost-analytic methods in telemedicine are briefly described in Appendix 1.

Among the half dozen types of cost evaluation used frequently in health-care services research, CEA is most prominent. As described in the appendix, whereas costs are monetary in a CEA, personal health-care outcomes are reported in nonmonetary terms, such as changes in blood pressure, or longer-term outcomes, such as improvements in quality-adjusted life years (QALYs), where years of life are weighted by health-related quality measures. ${ }^{4}$ A resulting program assessment metric such as "cost per QALY" provides a common currency that is especially helpful for comparing healthcare technologies. ${ }^{5}$

Of course, each cost and benefit method has advantages and disadvantages with implications for measurements of benefit, potential comparisons among alternatives, and types of outcomes that can be captured in the analysis. The different methods, as well as details in this implementation, determine the range and types of effects captured in the analysis, as well as the potential for comparisons among alternatives. For example, selecting episodes of illness as the outcome of a CEA evaluation limits the potential to identify the effects of telemedicine on preventing new episodes of illness. Using visits or virtual visits as the outcome ignores the potential of telemedicine to treat episodes with fewer provider contacts, or fewer hospitalizations. 
Cost minimization analysis (CMA) of telemedicine generally ignores any potential for telemedicine to affect health-related outcomes, because CMA strictly focuses on cost-related benefits of the technology.

In summary, understanding cost-benefit concepts applied to the economic theory of choice is fundamental to both appropriate design and interpretation of telemedicine cost evaluations. This affects the identification and measurement of appropriate inputs and outputs of telemedicine and its alternatives. The concepts and the selection of a cost and benefit method constrain the potential inference from and the interpretation of findings.

\section{Production functions in telemedicine cost evaluations}

Compared with the economic theory of choice, other areas of economic theory are far less pivotal in health-care program cost evaluation. However, economic concepts of production appear likely to contribute to the design of telemedicine cost evaluation. This theory also has the potential to spur the development of a body of telemedicine production knowledge and models with predictive content.

Berki first proposed the use of an economic production function to structure our understanding of telemedicine, stating " . . the fundamental effects flowing from the introduction of telemedicine will be changes in the process of production outputs as well as changes in the outputs themselves." 6 His detailed development of an economic production function of telemedicine was employed to generate insight and questions on the implications of telemedicine and the design of telemedicine economic research. In particular, he identified the need for greater specificity in types of telemedicine and their outputs when attempting to analyze telemedicine costs. He also identified the need to address telemedicine as being embedded within a health-care system.

Although not providing a predictive theory of costs, Berki emphasized the use of telemedicine production analyses to identify an extensive range of potential inputs and outputs. He also identified the complexity of cost research design and interpretation. Economic produc- tion theory provides a logical structure to identify and evaluate cost determinants, resources that affect costs, and the associated outcomes. It identifies both potential factors and logic related to the transformation from resources into such outcomes as access, health, and satisfaction. Hence, the body of telemedicine cost findings may be more easily understood through this framework, which, when combined with specific findings, may improve future research and lead to predictive models for telemedicine cost research.

\section{Cost assessment framework}

Biomedical devices may be described through a set of technical specifications; however, the content of a telemedicine program is less easy to define. Most often, telemedicine programs are described through areas of medical specialty or location of care. These attributes, however, are insufficient for specifying production inputs and likely outputs. Moreover, contextual factors of delivery programs, such as the boundary between teleradiology and standard radiology, are much more ambiguous. Consequently, additional descriptions of telemedicine programs are often needed. The question is what constitutes the program? What are the critical dimensions that facilitate more homogeneous groupings or adjustments for comparison of findings?

Some of the useful attributes for characterizing telemedicine programs are those that determine the use and value of program inputs or outputs, or that characterize assessments of opportunity costs. These attributes may be viewed as a cost assessment framework, which is not a well-defined algorithm or theory, but rather a description of a telemedicine program that is situationally relevant in identifying and defining costs and benefits. For example, such characterization reveals how a program is defined and the decision-making time horizon, both of which affect costs for the stakeholder. The decision maker's objectives, delineation of the boundaries between the intervention and context, and identification of a target audience for use of research results are major underlying assumptions that characterize the cost evaluation problem. 
The cost assessment framework as defined in Table 1 is closely related to components used in defining CEA by the United States Public Health Service (USPHS) Consensus Panel. ${ }^{7}$ The major dimensions of this framework help assess telemedicine cost-evaluation design and interpretation.

\section{Program objectives and study purpose}

A principal aim of telemedicine cost evaluation is to provide decision makers with information on costs of alternatives decisions. This requires an accurate understanding of objectives so as to obtain a precise identification and valuation of what is given up and gained as the result of an action. For example, a program with a short-term treatment objective will incur different costs and benefits than one focusing on prevention. Without sufficient knowledge of the decision-making objective, the empirical research and analysis may not be relevant. That is, precise evidence on the wrong question is most often not helpful. Consequently, it is often useful to have a pre-evaluation assessment of the decision maker's objectives. Careful consideration of objectives may be warranted when interpreting findings in the telemedicine literature.

Notably, the issue of program objectives is related to but separate from that of the decision maker's perspective. Different types of decision makers, such as the patient, provider, payer, and society at large, may have different objectives for a program and different values assigned to benefits. But these groups are also likely to incur different costs and benefits from the program, even if they agree on the same program objective.

Table 1. Cost Assessment Framework

A. Program/decision-making objective

B. Study purpose-constituency

C. Target decision maker

Perspective/stakeholder Audience

D. Program definition and boundaries

Type of analysis

Decision maker

Target population

Decision time horizon

Decision maker time preference
This difference in perspective results in different costs and benefits among the stakeholders, and sometimes may result in differing incentives for program participation. The appropriate perspective for an evaluation will depend on the purpose of the evaluation. On the other hand, many researchers advocate the inclusion of the societal perspective (where all program costs and benefits are included in the CBA, regardless of who incurs or accrues them) in reporting results. The intent here is to facilitate comparability across studies.

At the same time, the primary purpose of telemedicine cost evaluation is to support health-care policy and organizational decision making. For this purpose, a cost assessment framework may guide the evaluation, but for that evaluation researchers may also subscribe to a larger role of ongoing knowledge building by making study results usable for a broad population of stakeholders with similar objectives. Here, simply the intended scope in the generality of findings may affect the methods of evaluation. For example, an evaluation may provide valid and generalizable results at a local or national level. Variations in international prices, population preferences, and health system efficiency, however, may undermine crosscountry generalizations. ${ }^{8}$ The challenge becomes one of the research tradeoffs required to address contextual factors that influence the generality of results. Less powerful findings locally may be sacrificed for the power of broad generalization.

\section{Telemedicine program definition and context}

Telemedicine refers to a wide range of activities providing health care at a distance. These include remote monitoring, where capabilities are largely defined by device specifications; medical encounters that may be defined by enabling devices; and complex delivery programs, such as virtual hospitals that employ a complex mix of technologies and innovative processes. Thus, telemedicine interventions may include hardware and software technology, a clinical process, and a broad system of health-care delivery.

With device-oriented telemedicine, the definition of the program, its functional capabili- 
ties and resources, and the relevance of any contextual factors are more easily identified. In contrast, as telemedicine becomes a complex program of activities with different resources and multiple outcomes, the heterogeneity of telemedicine capabilities, capacities, and resources create a substantial challenge to be generalized from an evaluation. Closely integrated technologies, such as information technology systems and contextual factors, are likely to have dependencies in the system of care that are not easily separated from core telemedicine capabilities.

At the same time, telemedicine-oriented systems of care are more likely to provide needed economies of scale, joint gains from technology, and manpower knowledge efficiencies. In addition, the complexity of systems and activities related to telemedicine systems are likely to make program performance less determined by technical capabilities and increasingly dependent on management efficiencies and incentives, as has been discovered with information technology in general. ${ }^{9}$ Hence, the defining attributes of a promising telemedicine intervention are likely to change as applications shift from narrowly defined, technically oriented clinical telemedicine capabilities to reliance on systems of information technology with behaviorally defined production processes.

The result is that different approaches and concepts may apply to cost evaluation of device-dominant telemedicine as compared with system- or activity-dominant telemedicine programs. Basic comparisons of alternatives that fit the evaluation paradigm appear to be less appropriate for cost evaluations of less technologically dominant and complex telemedicine systems of care. The cost impacts of telemedicine systems may be determined by a set of contextual factors, some of which may be identified through economic theories of organization and competition.

\section{Methods of inference}

RCTs constitute the most powerful methodology for testing the impact of a medical intervention, procedure, or medication. Medicine has been well served by RCTs and cost-effectiveness/cost-utility evaluations of pharma- ceuticals and medical devices have been quite successful by employing RCT methods of inference. Nonetheless, whereas RCT methods may be good for evaluating telemedicine cost, other methods of statistical analysis may be warranted and even preferred.

In contrast to telemedicine, several areas of evaluation accept alternative methods for demonstrating causality though the rationale may not be without some debate. Of course, medical research has the strongest tradition of randomized experimental design for causal assessment, and perhaps because of this, nonRCT methods have had limited use in evaluating telemedicine costs. Several arguments for using alternative methods of inference appear credible in relation to program cost evaluation, including when:

- Threats to external validity transcend the RCT contribution to internal validity,

- Relationships between the intervention and context cannot be disentangled through random assignment,

- RCTs are not feasible or the additional confidence in outcomes provided by RCTs is economically justified,

- Question of interest is not an unbiased estimate of program costs in a population but an estimate of costs in an uncontrolled setting (i.e., when self-selection is allowed).

The strength of RCT derives from ensuring the internal validity of findings. ${ }^{10}$ But important determinants of costs, such as organizational competence, factor prices, and health system policy, are difficult to randomize, and they may have a more significant impact on cost than the intervention. Hence, the RCT findings from telemedicine programs cost evaluations may have limited value to decision makers who are concerned about the overwhelming effects of their context. Moreover, when the performance of telemedicine is determined by a system of variables, such as those involved in health-care production, or conditional on factors, such as managerial incentives, contextual effects may be impossible to disentangle from contextual factors. In neither case do RCT methods provide the best information for the decision. 
For some types of telemedicine costs questions, decision makers may not be interested in the unbiased estimates of costs. For example, a third-party payer may be interested in the cost of self-selection biased program, i.e., program costs when patients have self-selected. Selection and choice are important components in the design of a health-care delivery system, so unbiased cost-effectiveness may not be useful without knowing the cost bias of choice.

To summarize, the methodological question is to determine the appropriate or "cost-effective" methods of inference, matching the evaluation design to the evaluation's purpose, resources, and stage of development.

\section{Cost analysis guidelines}

Guidelines are increasingly available for conducting, evaluating, and synthesizing healthcare research. They provide a powerful tool for improving the quality and comparability of studies. However, although economic cost analysis is tied to an economic theory of choice, there is room for considerable latitude and differences of opinion in applications. For example, standardizing definitions would improve the comparability of studies. Errors and methodological limitations of cost analysis are reported throughout the literature. Nonetheless, guidelines are designed to improve the quality of research in this area.

Spurred by their potential to provide a significant collective value for health-care cost research, health-care cost analysis guidelines have evolved rapidly.,11 A few sources focus on assessing and improving quality of evaluation studies. ${ }^{12}$ Most notably, the Cochrane Collaboration provides guidance for systematized assessment and synthesis of study evidence. ${ }^{12}$ Other groups have developed guidelines that promote consensus or preferred definitions and methods for research, and less explicitly attempt to determine evaluative standards. At least 10 sets of formal and informal guidelines dedicated to health care cost analysis, mostly related to pharmaceuticals and devices, have been developed by government and industry organizations. Perhaps the most detailed of these are the USPHS Consensus Panel guidelines that demonstrate a particular interest im- proving the comparability of studies. ${ }^{7}$ The Consensus Panel provides a theoretically consistent cost assessment framework for: (1) defining a cost or resource allocation decisions, including decision-making objectives and defining the program or intervention; (2) determining the decision model of costs and benefits; (3) specifying data and measures; (4) performing appropriate statistical, modeling, and sensitivity analyses; and (5) reporting results understandable by decision makers and researchers.

Notable improvements in compliance with key guidelines have been reported since publication of the consensus panel recommendations. ${ }^{13}$ On the other hand, analysis of published cost analysis guidelines found agreement for about $75 \%$ of methodological aspects. ${ }^{3}$ Disagreement was found in the recommended choice of perspective, the types of resources considered, and costs that should be included in the analysis. Partly, the differences in the guidelines appear to stem from differences in research objectives and associated technical issues. As a recent assessment of evaluative systematic reviews concluded, "Methods for assessment of methodological quality by systematic reviews are still in their infancy, and there is substantial room for improvement."14

\section{IDENTIFYING AND SELECTING TELEMEDICINE COST EVALUATION LITERATURE}

The status of telemedicine cost research is shaped by research findings, methodologies, and the resulting conceptual understanding of telemedicine costs. To this end, this section offers an approach and selection process for obtaining an accurate representation of this literature.

Several authors have used formal guidelines in conducting systematic literature searches in reviewing of telemedicine cost evaluations. ${ }^{15-20}$ Typically, these searches have used topical keywords to identify articles, and then explicit criteria on scientific merit for including or excluding studies in the review, with the objective of providing an efficient and unbiased estimate of costs and benefits. This paper builds on these earlier works, but not simply by 
adding the recent months of findings. Taking advantage of these earlier efforts, the results of earlier reviews and key studies are combined with newly identified studies. The emphasis of this assessment is on the conceptual and methodological rigor in cost studies, on the usefulness of identified cost issues, on research methods and strategies, and on conceptual interpretation. The concepts and methods discussed in previous sections of this paper ${ }^{21}$ are used to guide the following search, selection, and review.

Still, identifying the boundaries for selecting articles remains a formidable task, which is especially apparent in terms of the conceptual ambiguities surrounding telemedicine costs. Activities that may be considered telemedicine continue to blend applications of information and communications technologies. Of course, the history of telemedicine research has been characterized by substantive debate on definitions, and this continues to evolve with technology. As a result, the use of a defined set of telemedicine keywords in selecting literature may easily omit relevant studies, but the most ambitious expansion of terms begins to capture an unmanageable number of studies.

An efficient alternative for selecting and reading abstracts produced by the most expansive set of keywords evolved from first considering studies identified through previous systematic reviews and then using keyword and database selection strategies to augment the literature. This approach may be best defined as iterative triangulation. That is, first previous reviews and standard initial sets of telemedicine keywords were used to create a database of abstracts for review. These were used to create a database of keywords, which resulted in overlapping sets of literature. Sets of prominent and seemingly relevant keywords were examined to identify additional articles, but investigating the detailed subsets of articles depended on the initial payoff in the discovery of new articles.

At the same time, significant references were also identified from the lists of references in the original articles. While the primary focus was on telemedicine cost literature, other prominent databases in the literature were included, such as health-care technology assessment
(HTA) and health-care information technology (HIT) (Table 2). These searches were guided largely by the need for further considering significant issues in the telemedicine cost literature.

The database of resulting articles consisted of studies identified through electronic searches and manual scans of both published and unpublished studies, resulting in a database of 1,430 telemedicine articles (Table 3). Abstracts for each of these were reviewed. Among these articles, abstracts that indicated cost analysis with empirical data, or appeared to have rigorous or unique cost analysis research methods were selected for further consideration.

Finally, a database of studies based on standard telemedicine keywords, related analytic area keywords, and all related articles was constructed. Software was used to create and map clusters of keywords, which were inspected to identify unrevealed telemedicine related groups of studies. Clustering analyses of keywords associated with over 9,000 articles were evaluated to establish consistency across selection methods and significant omissions in topical and methodological areas of telemedicine costs. This resulted in the identification of few additional papers, but no new topical or methodological areas were added to the analysis.

\section{AN ASSESSMENT OF RESEARCH FINDINGS AND METHODOLOGIES}

The prevailing consensus is that few telemedicine cost studies have provided eco-

Table 2. Keywords and Concepts Used in Search

\begin{tabular}{ll}
\hline Primary terms & \multicolumn{1}{c}{ Secondary terms } \\
\hline Telemedicine & Cost \\
Telecommunications & Cost allocation \\
Telehealth & Cost analysis \\
Telemedicine & Cost benefit analysis \\
Telenursing & Cost effectiveness \\
Telepathology & Cost minimization \\
Teleradiology & Cost savings \\
Telemetry & Health-care costs \\
Telephone & Health information technology \\
Television & Technology assessment \\
& Methods and methodology \\
& Economics \\
\hline
\end{tabular}


Table 3. Searched Sources of Literature

\begin{tabular}{|c|c|}
\hline Type of source & Sources \\
\hline Published literature & $\begin{array}{l}\text { NLM Databases-MEDLINE (1/1976-3/2004); HSAT } \\
\text { EMBASE (1/1995-3/2004) } \\
\text { ISI Science Citation Index Expanded } \\
\text { ECO-An Online Computer Library Center (OCLC) collection of scholarly journals, } \\
\text { CINAHL-Cumulative Index to Nursing and Allied Health Literature } \\
\text { JEL-Journal of Economic literature }\end{array}$ \\
\hline World Wide Web & $\begin{array}{l}\text { AHRQ: http://www.ahcpr.gov/ } \\
\text { NHS Health Technology Assessment Programme: http://www.hta.nhsweb.nhs.uk/main.htm } \\
\text { NHS National Institute for Clinical Excellence (NICE): http://www.nice.org.uk/ } \\
\text { Netting the Evidence: http://www.sheffield.ac.uk/ scharr/ir/netting } \\
\text { Canadian Coordinating Office for Health Technology Assessment (CCOHTA): } \\
\text { http://www.ccohta.ca/ } \\
\text { Office for the Advancement of Telehealth: http://www.telehealth.hrsa.gov/ } \\
\text { VHA Telehealth: http://www.va.gov/telehealth/ } \\
\text { Telemedicine Information Exchange: http://tie.telemed.org/ } \\
\text { AHRQ Evidence Based Practice Centers } \\
\text { Cochrane technology databases } \\
\text { McKinsey Global Institute: } \underline{\text { http://www.mckinsey.com/knowledge/mgi/ }}\end{array}$ \\
\hline Conference reports & $\begin{array}{l}\text { HIMSS conference reports: } 1995-2003 \\
\text { AMIA conference reports }\end{array}$ \\
\hline $\begin{array}{l}\text { Hand-searched } \\
\text { journals }\end{array}$ & $\begin{array}{l}\text { Journal of Telemedicine and Telecare } \\
\text { Telemedicine Journal/Telemedicine Journal and e-Health } \\
\text { Journal of Health Economics } \\
\text { Health Economics } \\
\text { Value of Health } \\
\text { Journal of Healthcare Information Management } \\
\text { International Journal of Technology Assessment in Health Care } \\
\text { Journal of Information Systems }\end{array}$ \\
\hline
\end{tabular}

nomic results that are useful for organizational or policy decision making in telemedicine. ${ }^{15,22}$ Research reviewers have consistently noted a small number of useful findings, ${ }^{15,22,17,23}$ and even these are marked by questionable use of standard cost techniques. Hence, they contribute little to the cost evidence. ${ }^{15,24,25}$ Similarly, but perhaps having a different perspective on what is lacking, providers and payers continue to express a need for cost evidence for decision making.

Analysts have provided many explanations for the perceived inadequacy of telemedicine cost information. Studies have been faulted for having small user populations or data, fledgling programs with premature evaluation, biases due to reimbursement policy, faulty economics, and inadequate research methodology. ${ }^{18,26}$ In particular, the limitations of the literature have been most commonly attributed to inadequate and insufficient use of RCTbased studies. At the same time, at a more fundamental level, telemedicine cost research has been viewed as being encumbered by its "complex mix of technology and services." 15 This review considers whether current telemedicine cost evaluation concepts and methods constitue a barrier to useful cost research, and whether uses of economic theory and alternative research methods would improve the value of output from telemedicine cost research. Assessments are provided through discussions of the findings and limitations of telemedicine cost evaluation research.

\section{An economic interpretation of cost findings}

Reviewers of telemedicine cost studies bemoan the inadequacy of research methods and the resulting small number of useful cost findings. This conclusion is partly driven by the standards of methodological orthodoxy, or rigidity, for causal analysis. But, it also depends on assumptions about how findings reflect on the specific cost problem. In particular, RCT methods are especially appropriate for discrete, 
well-specified interventions. Accordingly, the research design has been to address different types of telemedicine as distinct technologies. This specification has been maintained in the interpretation and integration of findings. The notion of discrete interventions has been translated as conceptually independent of the different types of telemedicine findings. Often, the assumption of conceptual independence of telemedicine program cost findings is unnecessary.

The economic underpinnings of cost evaluations may provide a basis for the conceptual integration of the seemingly disparate telemedicine cost results. In particular, the interpretation of cost results from telemedicine programs may make use of the economic production concepts previously discussed. Specifics on the approach used in a cost analysis may be combined with other knowledge of medical care delivery and economic production to synthesize findings around a distinct cost principle. Accordingly, the following sections attempt to make more efficient use of telemedicine cost research by assessing how they reflect on economic processes that determine costs.

\section{Cost impacts on access to a medical visits}

The earliest studies of telemedicine costs concentrated on the cost implications of enabling access to care. As such, studies primarily considered the costs or economic gains from reductions in patient or provider time traveling for care. These studies often involved clinical technologies that were considered unchanged when provided through telemedicine. For example, for purposes of cost evaluation, the clinical character of a radiological service or other imaging service was considered to be essentially identical to in-person service.

Early cost studies often implicitly or explicitly were presented as cost-minimization studies, but they were more unique than that. The basic specification of the problem in these studies was to limit the telemedicine effect to the production of access while defining the cost structure as a trade-off between gains from reduced travel costs and expenditures on Information and Communications Technology (ICT) and personnel. Hence, high barriers to access and specialties requiring less expensive technologies were likely to produce favorable results for telemedicine.

Using this approach, telemedicine produces cost savings for specific populations, such as prison inmates, ${ }^{26,27}$ sailors at sea, ${ }^{28}$ or emergency cases..$^{29,30}$ Hence, the potentially prohibitive determinant in the use of care is the cost of travel, which can be offset by efficient use of the ICT technology. Specialties that required cheaper technologies, such as psychiatry, or that easily integrated the technology in the visit, such as dermatology, were more likely to show savings through reduced travel costs. On the other hand, these studies were important only to decision makers who incurred a sufficient saving from travel costs. Most notably these included U.S. prisons, the military services, and a number of European health-care systems.

Of course, a reasonable conclusion is that once the technical feasibility for telemedicine is established, the potential for savings from travel for patients would be expected. The evidence suggests that travel-based cost savings are feasible for a variety of telemedicine specialty applications, including teleradiology, teleneurology, teledermatology, and teleoncology. ${ }^{29-32}$ However, the findings for other types of telemedicine, such as telepsychiatry, are inconsistent. This may be due to the fact that some of these study designs focused on the impact of telemedicine on access, whereas others included the effect of telemedicine on clinical efficiency. The latter is the subject of discussion in the next section.

Clinical efficiency aside, the expected savings in this type of telemedicine analysis are situationally dependent; that is, they depend on the environmental context, such as general costs of travel and technology. More importantly, once feasibility is established, significant economic findings are not likely through a local single-site clinical trial. The key parameters affecting costs, location, and price of technology are external to the design of local clinical trials.

\section{Costs impact on clinical efficiency}

A second category of telemedicine programs effects may be characterized as changing the 
costs of care by affecting clinical efficiency, as well as travel and technology. But here telemedicine may result in changes in the amount or mix of resources and thereby the costs of clinical care. Changes in clinical care processes decrease or increase cost care. This may best be reflected in assessing episodes of care.

This additional role of telemedicine in affecting costs is significant from many perspectives. First, clinical costs often have a dominant share of total costs of care, especially for populations without exceptional barriers in access. Consequently, efficiency savings through telemedicine present a much greater opportunity for finding savings. These clinical efficiencies may be influenced more by patient and physician characteristics and less by local situational factors. Moreover, and perhaps as important, depending less on local factors allows findings on the cost impact telemedicine to be applied more easily to other settings. Presumed costs savings due to the effects of telemedicine on the production process of medical care are more likely generalizable across settings.

Analyses of telemedicine that capture the costs impacts of clinical efficiencies may be defined implicitly or explicitly through several features of the study design. First, the analyst may explicitly recognize the change in the mix or types of resources used in the clinical process. For example, store-and-forward dermatology is likely to be basic change in the clinical process when compared with real-time teledermatology. Alternatively, many studies implicitly allow for impacts on clinical efficiencies through their definitions of the output. For example, where the cost impacts are examined across multiple patient encounters or visits, then telemedicine may affect clinical inputs. The number or mix of clinical visits may be changed in the treatment of the care.

Studies of teledermatology may best illustrate the merit of this conceptualization in guiding the integration and interpretation of findings. Dermatological conditions often occur in relatively well-differentiated episodes. Consequently, telemedicine studies frequently link multiple provider encounters. At the same time, dermatology practice lends itself to the use of alternative sets of resources in the production of a telemedicine visit, such as real- time and store-and-forward processes of care. The result is that each of the three sources of cost impacts may apply. But changes in the clinical process for real-time teledermatology are less likely to affect total costs, as compared with store-and-forward processes.

Studies of teledermatology fit surprisingly well within the constructed cost framework. The cost savings of real-time and store-andforward teledermatology depend on the costs of travel. But store-and-forward costs have proven to be more variable across studies and sensitive to specific assumption about the production of clinical care, such as patient flow. Moreover, although an RCT study of a Veteran's Health Administration teledermatology program found it not to be cost effective, further analysis of the results indicated that it became cost effective with modest changes in clinical or travel costs. Accordingly, costs of a teledermatology program are likely to depend on the situational and generalizable impacts of telemedicine, and that decision makers need to consider the relative roles of each. At the same time, researchers may contribute by further analyzing the determinants of clinical productivity in teledermatology and the relative magnitude of these when compared with the savings from travel and the costs of technology.

Telepsychiatry presents a contrasting set of insights when examined through this conceptualization of the sources of telemedicine costs. Telepsychiatry has been shown to be feasible, may increase access to care, and often is reported cost effective. The unit of output in telepsychiatry is the visit, but this is somewhat ambiguous as a measure of treatment output. At the same time, there is considerable latitude in the resources used to provide telepsychiatry. Moreover, telepsychiatry has lacked standard models or guidelines for care, although several detailed guidelines have been recently published.

Consequently, it becomes difficult to determine how much or whether telemedicine changes the productivity of the psychiatric clinical process. Telepsychiatry is usually reported as less expensive for patients when the cost of travel and lost time are considered. ${ }^{33}$ This is especially apparent when care is provided to prison populations, remote clinics, and other- 
wise isolated populations. ${ }^{34}$ Indeed, telepsychiatry services have been estimated to be less expensive, as expensive, or more expensive than in-person services. ${ }^{35}$ The small size of many programs may be a problem, and some approaches to the delivery of telepsychiatry have been reported be more cost-effective than others. ${ }^{33}$

The implication of this economic interpretation of telemedicine findings is that telepsychiatry may be cost-effective, but the findings are unclear. Certainly, RCT studies may provide better evidence, but measurement issues appear to be a major barrier to drawing definitive conclusions. Future studies of telepsychiatry should focus on telepsychiatry outputs and the changes in the mix and use of resources in the clinical care process.

\section{Cost impacts on disease management}

Some telemedicine programs may be viewed as having a direct effect on the process of maintaining health and well-being (referred to by economists as the production of health) rather than simply affecting access and efficiency. Economic analyses from outside of telemedicine have indicated that activities that prevent illness are often far more cost-effective than medical care treatments. ${ }^{36}$ Accordingly, telemedicine interventions that reduce the use of medical care through primary or secondary prevention potentially could save costs that might overshadow the effects on access alone. The costs saved through telemedicine through reduced travel to medical care needed in the absence of the preventive activity likely would pale in comparison.

Home telecare and telemonitoring may offer significant cost savings in terms of health and wellness. These applications may provide little improvement in access to clinical treatment, and they may not have a significant effect on the cost-effectiveness of clinical care. But they can change the need for care. Recent evidence suggests that significant savings are possible through the use of telehealth to diminish the need for medical care. ${ }^{37,38}$ For example, a randomized controlled trial of prenatal telehomecare for high-risk pregnancies reported a reduction in hospital use. ${ }^{39}$ Subsequently, a landmark RCT study of home telecare demonstrated cost-savings through reduced utilization. ${ }^{38}$ In this latter study, remote monitoring and real-time video interactions reduced total costs of care relative to those receiving traditional homecare. More recent studies further provide evidence of the potential cost-effectiveness of telemonitoring among congestive heart failure and diabetic patients. ${ }^{37,38}$

Overall, a notable contrast has emerged in the apparent success of telemonitoring and home telecare cost-effectiveness studies, when compared with the limited results from teleconsultations. ${ }^{19,40,41}$ A number of explanations for this difference seem plausible. Home telecare and telemonitoring occur outside established medical facilities and are therefore less likely burdened by historical overhead costs that may or may not be necessary for other types of telemedicine. This application may represent a more targeted intervention of highrisk patients with a narrowly defined technology. These findings are likely to generate even more high-quality research in this area.

On the other hand, it may also be, as some economists have long argued, ${ }^{36}$ that more medical care may not be as cost-effective as maintaining or improving health. In this case, home telecare may be benefiting from the underlying opportunity in the production of health. If so, telemedicine applications that further exploit investment opportunities in wellness would have a higher likelihood of success.

\section{The contextual assessment of cost findings}

Health economics concepts, such as productions functions, may improve the design and interpretation of telemedicine cost analysis. Yet, the interpretation and synthesis of cost evaluations also requires an understanding of the decision-making assumptions and environmental context of the telemedicine activities that incur costs. Here, this is defined as the contextual assessment of the cost evaluation and includes both the decision-making context as represented in the "Cost Assessment Framework" and the environmental context of the telemedicine program that may impact its performance.

Cost evaluation findings on a telemedicine activity depend on such decision-making 
specifics as the perspective, the target population, and the decision time horizon. Contextual factors, such as provider fee levels, equipment costs, and health system efficiencies, may determine the external validity of findings. Although both the decision-making and environmental context of findings may limit the applicability of findings, an understanding and even separate analysis of these factors may facilitate further synthesis of disparate findings. For example, adjustments to local provider costs may provide insights into observed differences in study results.

This section examines issues pertaining to contextual determinants of cost findings and ways in which understanding the context facilitates the interpretation and synthesis of results. Also, some research strategies that may address these contextual factors that can improve telemedicine cost evaluations are considered.

\section{Decision maker and information requirements}

Existing telemedicine cost research and analyses rarely discuss the decision-making objectives that would be supported by the results of a specific study. Neither do they explicitly define the informational needs of decision makers who would use such results. Nonetheless, each of these is likely to have profound effects on the design of the study, methods of statistical inference, and the interpretation and usefulness of study results.

Many evaluations performed in the United States appear to define the research question so as to obtain cost-effectiveness information needed for directly funded government healthcare delivery programs. But these seldom translate easily to the decision-making needs of market-driven institutions and third-party payers. For example, researchers frequently rely only on the societal perspective in evaluating costs and benefits. All costs and all benefits are considered regardless of who pays the cost or gains the benefit. However, private health-care institutional decision making will be based on a narrower set of costs and benefits, often, within the context of a business case that considers the costs and benefits related to institutional expenditures and revenues. A third-party insurer will likely consider the costs and benefits quite differently. An insurer is likely to be especially interested in the effects of a reimbursement policy on its costs and revenues.

At the same time, and perhaps inconsistently, the research designs in economic studies of telemedicine appear to focus on local decision making, and do not address contextual variables and measurements that facilitate applications in other settings. Hence, it is incumbent on the analyst of truly generalizable research to address issues of comparability of costs and benefits, external validity, and relevance of research for various health-care decision-making or policy objectives.

At the same time, this does not imply that a locally focused evaluation is of low quality, especially if it provides information needed for decision making. The approach and findings limit their range of applicability. For example, differences in unit costs of inputs have been found to affect CEA ratios among European Union countries. ${ }^{42}$ An analysis by Drummond also led him to conclude that it is difficult to infer CEA results between countries. This problem becomes even more difficult if countries vary in the resources used to produce a given result. ${ }^{43,44}$

The dilemma is that costs and benefits of research also have to be considered, and generalization has a cost. The optimal trade-off partly depends on the specific needs of decision-makers and the expected scope of generalization of results.

\section{Complexity of telemedicine interventions}

Evaluating the costs and benefits of telemedicine depends on identifying the resource basis used in producing telemedicine. Costs and benefits may be defined largely through the devices used, as in some telemonitoring. They may be affected by various factors, including sharing the technology for other functions and an array of work processes and external organizational interactions, as in a communitywide telepyschiatry program. Yet, precisely how we specify the intervention sets the challenge in measuring costs and benefits. For example, digital radiography is often cost-effective separate from it use in telemedicine. It 
provides savings in the reduced cost of film and related supplies as well as through fewer lost images. But once the technology for digital radiography is available, the cost of the additional systems to implement teleradiology is dramatically reduced. Of course, the benefits also become limited to gains from the electronic exchange of radiography. Nonetheless, despite increasingly widespread acquisition of digital imaging systems, there appears to be no comprehensive estimate of the economic returns on investment. 45

The definition that distinguishes the intervention from context may also delineate the potential generalization of findings. For instance, a narrowly defined intervention, such as a telemedicine device, may be more consistently identified, characterized for costs and benefits, and reproduced in new settings. Modifications or adjustments, such as in prices, may be needed to apply results in a new context. These are less likely to be a problem for decision makers. In contrast, the greatest challenge in applying economic evaluation methods is in programs that have ambiguous boundaries of intervention and broadly defined or intangible outputs. ${ }^{46}$ For example, it would be far more challenging to identify clearly inputs and associated input costs and to track outcomes in a broadly defined telehealth network. Similarly, programs that rely on extensive and complex interactions imbedded in organizational processes present difficulties in measuring costs and outcomes. On the other hand, telemedicine interventions that deliver the greatest cost savings likely to be narrow and well defined, such as monitoring of congestive heart failure patients, or home telecare. ${ }^{37,38}$

\section{Methods for statistical inference}

The study designs employed in economic evaluations include decision analytic models, observational studies, and clinical trials. Observational studies are more prevalent in the literature. Prior to the mid-1990s, health-care cost analyses often used clinical trial data on effectiveness to develop a decision model for determining costs and benefits. Within the last decade, several cost studies have been integrated with RCTs, but the majority do not do so. ${ }^{47}$
Perhaps this evolution explains the apparent preference for RCT or quasi-experimental designs, and the growing number of RCT-based studies. Nonetheless, these studies tend to rely on comparative evaluations of program outcome measures, and are mostly patterned after the clinical trials of medical care research. The presumed power of the RCT is that randomization of patients to telemedicine and traditional care settings assures unbiased comparisons in the use of resources and outcomes. This is certainly the case when evaluating devices such as tele-EKGs. Still, the requirements of some research may suggest the use of alternative methods of inference, either in combination or as an alternative. This is because telemedicine interventions are often complex systems of devices and activities, the performance of which significantly depends on contextual factors that are independent of the research treatment.

RCT designs in telemedicine economic research would be subject to significant external validity threats from economic determinants not controlled through randomized assignment of patients. ${ }^{48}$ For example, telemedicine interventions may involve extensive process and behavioral changes that are affected by health-care policy, local resources costs, resource costs, and incentives that are beyond the boundary of the research randomization. Despite the use of randomized, controlled trials, the systemic nature of the telemedicine production process may easily be affected by major contextual factors that will limit external validity of findings. Alternative, non-RCT processes and concept models may be available to reduce error variance and biased results. Selectivity models that better address beneficiary behaviors and policy maker needs when faced with new technologies have proven useful in other areas of health-care research. Techniques that combine trial-based and observational data, sample selection, and censored data estimation are among current major development across areas of health-care cost analysis. ${ }^{48}$

Consequently, the selection of the most appropriate methods of inference in telemedicine cost research depends on a range of multivariate statistical techniques to improve the efficiency of estimation and adjustment for selec- 
tion bias. These approaches may be used as alternatives or complements to RCT methods, especially as a means of revealing logical connections among inputs, context, and outputs and in leveraging and the generality of findings.

\section{SUMMARY AND RECOMMENDATIONS}

Lamenting the lack of information on telemedicine cost analysis has become a standard preface to discussions of telemedicine evaluation. An ample volume and variety of explanations for the dearth of results are often cited. These include small user populations, rapidly changing technology, poor selection and application of statistical and economic methodologies, and inherent failings in available economic evaluation methodologies. ${ }^{15,18,26}$

Still, researchers express a growing optimism in the value of telemedicine while government and health-care decision makers from many countries increasingly invest in telemedicine. Assuming rational behavior among the stakeholders, the evidence from these sentiments suggests that more convincing data on the costs and benefits of telemedicine exist. The incongruity of formal assessments and decisionmaker behavior suggests the need for a more careful design and assessments of current programs. The lack of convincing evaluations partly may reflect inadequate analytic assessment, integration, and synthesis of available data.

Cost evaluations of telemedicine have been undertaken in a diverse and global set of health-care delivery settings. Studies represent an economically heterogeneous set of programs and systems of care analyzed as discrete intervention alternatives. As a result, study data represent a large number of observations, often with little apparent commonality, and the integration of findings is arduous at best. A few notable findings have been identified, but results are meager for any specific technology or decision problem, and they are unlikely to provide much useful information. Yet, little attention has been paid to the unique methodological considerations and potential strategies for program cost evaluations. Economic and deci- sion-making concepts provide a host of tools to interpret and synthesize findings that appear to have been overlooked. These provide added insights into telemedicine program cost results once integrated meaningfully.

This paper was aimed at understanding available cost data, not simply from an assessment of the aggregate validity of statistical estimates. Statistically valid findings are limited. An attempt was made to use economic theory to understand the fragmentary and sparse findings. Nonetheless, an interpretation within an economic theoretical structure and applied decision-making framework provides additional corroborating evidence on the efficiencies and potential value of telemedicine. At the same time, these assessments provide insights as to potential design of telemedicine programs and further cost analysis research.

This review of telemedicine cost studies supports previous conclusions on the potential net savings to society through certain uses of telemedicine. Populations in remote areas, in prisons, or on ships may have reduced total cost of care by accessing it through telemedicine. But these reported gains in the cost-effectiveness of telemedicine depend on the reduced cost of access to care.

Distances and other barriers make the cost of travel to obtain care inordinately high, and the change in output with telemedicine has been along the dimension of access as opposed to changes in health. The relatively lower cost-tobenefit performance of these programs depends on changes in situational variables, such as geography, as well as reductions in the cost of information technology. Perhaps most importantly, savings from the relatively favorable cost performance of these programs may not accrue to the payers of care. Hence, the health plans on Europe, Canada, the U.S. Department of Defense, and prisons that pay for access may have incentives to provide this type of telemedicine. Otherwise, most U.S. plans do not pay the costs of barriers to access, and in fact may gain from these barriers.

On the other hand, generalizable telemedicine savings from clinical care efficiencies, as opposed to access savings, may be observed in studies of specialty areas that have a potentially modifiable clinical production process through 
telemedicine and that have a research design that allows for the detection of the potential gains. Cost evaluations are more likely to detect improvements in clinical efficiencies if outputs are at least measured as episodes of care. Both dermatology and psychiatry appear to have the potential for clinical efficiencies through telemedicine, but this is more clearly accomplished in store-and-forward dermatology where the process of care is most clearly changed. In telepsychiatry, changes in processes of care to take advantage of telemedicine are less clearly measurable.

The greatest potential for cost savings from telemedicine appears to be in its application in the production of health or wellness. When study outcomes are measured as health maintenance or wellness, as is usually done in home care, potential savings from telemedicine appear to be much greater. In particular, this type of telemedicine may be especially cost effective for high-risk and specific types of chronically ill patients.

The application of the economic concepts of production and contextual analysis and synthesis of cost-evaluation methodologies and findings appear to make better use of the cost literature. But the use of economic production concepts and the cost-assessment frameworks and the environmental determinants of program performance may make their greatest contribution in helping to define more innovative future research. Areas of likely payoff are studies of self-selection into telemedicine programs, adjustments for local health-care costs and system efficiencies, and the impact of incentives on specialties such as telepsychiatry where potential but difficult-to-measure changes in clinical efficiencies may occur.

Generally, the need to integrate current costs findings seems evident, and greater reliance on economic and decision-making structures seems desirable. Telemedicine technology is frequently considered a "black box." But telemedicine systems of care have important behavioral determinants and effects that may both better understood and evaluated through economic and health-care delivery concepts and through alternative approaches to assessing causality. Although the specific choice of methods of inference and design depend on specific research study needs and constraints, more flexible methods of establishing causality often may be justified and sometimes needed so as to aid interpretation and generalization of findings. Hence, a strict reliance on randomized, controlled trials for cost analysis is questionable.

Finally, telemedicine cost evaluation occupies a difficult divide between evaluation having a focus on providing information for local decision makers, and a focus on providing generalizable estimates of cost impact. General findings are needed to improve effectiveness and reduce costs to the broader community of decision makers; however, these likely have research costs and trade-offs that may not immediately benefit a researcher's constituency.

Consensus guidelines and best practices on costs provide common benefits to cost research, but they are unlikely to be supported by individual research or provider groups. Both the support and expansion of the common research contributions provided by guidelines and the development of strategies for addressing the balance of the private and shared-cost research costs and benefits question may make the greatest single contribution in enhancing the value of telemedicine cost literature.

\section{APPENDICES}

\section{Appendix 1: Methods of health-care cost analysis}

Cost-benefit analysis (CBA): CBA remains the paradigm for conceptualizing cost analysis, and it continues to serve as the ideal in defining opportunity costs and the valuation of benefits. In classic cost-benefit analysis, all input costs and benefits are transformed into monetary terms, such as dollars, as would be the case for individual decision-making in the market. The operational impediments to CBA are well documented for problems dealing with personal health-care services where the most significant issue may be the lack of generally accepted methods for translating benefits into dollars. Although various willingness-to-pay methodologies have been used for CBA of health services programs, ongoing public and economic debate over their use makes them unappealing. ${ }^{49}$ 
Cost-effectiveness analysis (CEA/CUA): The development of CEA was stimulated by the discomfort in CBA when translating medical outcomes such as years of life or a change in blood pressure into monetary values. Whereas costs are monetary in a CEA, outcomes are reported in nonmonetary terms. In personal health-care, these terms are expressed in such intermediate metrics as the outcomes of blood pressure or recidivism or such longer-term outcomes as quality-adjusted life years (QALYs), where a quality year may be partially defined by health. 4,7 A CUA is particular type of CEA that uses QALYs as an outcome. The resulting cost per QALY provides a common currency that is especially helpful for comparisons among disparate technologies. ${ }^{5}$

The objective in a typical CUA is to estimate the number of quality-adjusted life years produced through monetary expenditures on specific health-care interventions. QALY effects of an intervention are captured in the denominator of the ratio, whereas changes in resource use for an intervention are captured in the numerator in monetary terms. A result, for example, may be that a program costs $\$ 10,000$ per each additional life year. CUA ratios may be compared among a few alternatives or compared with a standard set of interventions, as from a league table that lists the cost per life year for a wide range of projects. ${ }^{50}$ Guidelines or "rules of thumb" for accepting interventions with CUA ratios, for example, of less than $\$ 100,000$ per life-year, have been a subject of extensive health-care research. ${ }^{24}$

The Panel on Cost Effectiveness in Health and Medicine (United States Public Health Service) advocates use of the CUA form of cost-effectiveness analysis. ${ }^{4}$

Cost-minimization analysis (CMA): Cost-minimization analysis (CMA) is essentially a costsavings analysis. That is, the focus of the analysis is on finding the alternative with the lowest cost; for example, the cheapest way to produce a medical visit or to treat a case. To the extent that telemedicine may not change an immediate health outcome and constitutes only a change in process, then use of CMA is justified. On the other hand, if benefits do change, information is lost and important findings may be overlooked when using CMA rather than CBA or CEA.

Return on investment (ROI): In its narrowest definition, return on investment is defined as a mathematical relationship, where the total monetary benefit from an investment is divided by initial and subsequent costs. ${ }^{47} \mathrm{ROI}$ is then stated as a percentage or ratio, and it provides a concise summary of factors that define the gains from an investment. As a good comparative summary of the profitability of an investment, this metric is especially well suited for for-profit organizational decision making. Yet, ROI is not usually cited or discussed in health-care economic cost research because it is viewed as organizational expenditures as opposed to opportunity costs, and its traditional methods do not include quantifying nonmonetary costs and benefits. Moreover, the ROI problem has been focused on capital investments for production, as opposed to consumption goods. That is, the organizational decision maker's interest is on the firm's monetary returns from an investment as opposed to the total value of benefits provided to a consumer.

Nonetheless, the term ROI increasingly is being applied to all cost-and-benefit methods. ${ }^{51}$ The broader definition encourages a comparison of an investment's total value and costs, including the consideration of nonmonetary benefits. ${ }^{47}$ For example, U.S. government guidance on information technology investments now defines ROI as encompassing the collection of quantitative cost/benefit methods, including CEA. ${ }^{47}$ But this question on the definition of ROI may not be clear, but broadening the concept has extended the focus of government analysts concerned with organizational decisionmaking tradeoffs, benefits, and costs. Then, while these government organizational expenditures still may not represent opportunity costs from the societal perspective, they represent opportunity costs within the framework of an organizational budget. Moreover, resulting cost analysis approaches may be applied equally to incorporate nonmarket and opportunity cost concepts in private business organization decision making, especially analyses and policies of private health-care organizations. 
Functional economic analysis (FEA): FEA is a variation of cost analysis developed to accommodate the needs of information technology and organizational decision making. ${ }^{52,53}$ Information technology products, including telemedicine, may be defined as sets of functions or capabilities as opposed to hardware, software, or a physical system. ${ }^{53-55}$ FEA focuses on the costs of the functions or capabili- ties that facilitate specific health-care objectives, such as providing medical care services or improved access to care. Thus, costs and benefits are associated with specific functions or information capabilities, such as providing an electronic medical record capability. Organizations may define decision-maker responsibilities and workflows that support functions to enable identification of costs.

Appendix 2: Comparison of cost evaluation methods

\begin{tabular}{|c|c|c|}
\hline Method & Characteristics & Disadvantages \\
\hline $\begin{array}{l}\text { Cost-benefit analysis } \\
\text { (CBA) }\end{array}$ & $\begin{array}{l}\text { Paradigm for cost analyses } \\
\text { Defines opportunity costs } \\
\text { Optimizes social welfare } \\
\text { Broad comparisons possible }\end{array}$ & $\begin{array}{l}\text { No generally accepted methods of } \\
\text { quantifying many benefits as dollars } \\
\text { Reluctance to express value of health or } \\
\text { life in monetary terms }\end{array}$ \\
\hline $\begin{array}{l}\text { Cost-effectiveness } \\
\text { analysis/cost utility } \\
\text { analysis (CEA/CUA) }\end{array}$ & $\begin{array}{l}\text { Outcomes reported in non-monetary terms } \\
\text { Outcomes may be expressed as QALYs } \\
\text { Preferred by U.S. Public Health Service } \\
\text { consensus panel } \\
\text { Benchmarks and comparisons available in } \\
\text { major online databases (http://www. } \\
\text { hsph.harvard.edu/cearegistry/) }\end{array}$ & $\begin{array}{l}\text { Inability to compare findings across } \\
\text { health-care areas. } \\
\text { Baseline } \\
\text { Generalizability of utility estimates } \\
\text { Costs of collecting utility data } \\
\text { Findings indicating that utility data } \\
\text { usually does not change a result }\end{array}$ \\
\hline $\begin{array}{l}\text { Cost-minimization } \\
\text { analysis (CMA) }\end{array}$ & $\begin{array}{l}\text { Lowest cost option sought } \\
\text { Less demanding data requirements }\end{array}$ & May overlook changes in benefits \\
\hline $\begin{array}{l}\text { Return on investment } \\
(\mathrm{ROI})\end{array}$ & $\begin{array}{l}\text { Mathematical relationship: monetary } \\
\text { benefit divided by initial and } \\
\text { subsequent costs } \\
\text { Facilitates investment analyses }\end{array}$ & $\begin{array}{l}\text { Better suited to for-profit decision making } \\
\text { Overlooks nonmonetary benefits } \\
\text { Focuses on capital investments for } \\
\text { production, not consumption }\end{array}$ \\
\hline $\begin{array}{l}\text { Functional economic } \\
\text { analysis (FEA) }\end{array}$ & $\begin{array}{l}\text { Facilitates analyses of information costs } \\
\text { and benefits } \\
\text { Benefits and costs derived for information } \\
\text { capabilities and functions }\end{array}$ & $\begin{array}{l}\text { Need for conceptual development of } \\
\text { functions } \\
\text { Generalizability }\end{array}$ \\
\hline
\end{tabular}

\section{REFERENCES}

1. Cameron AE, Bashshur RL, Halbritter K, Johnson EM, Cameron JW. Simulation methodology for estimating financial effects of telemedicine in West Virginia. Telemed I 1998;4:125-144.

2. Quinn-Patton M. The debate about randomized controls as the gold standard in evalution. National Institutes of Health, Videocasting: past events, http://videocast. nih.gov, 2004.

3. Hjelmgren J, Berggren F, Andersson F. Health economic guidelines: similarities, differences, and some implications. Value in Health 2001;4:225-250.

4. Garber A, Torrance G, Kamlet M. Theoretical foundations of cost-effectiveness analysis. In: MR Gold, JE Siegel, LB Russell, MC Weinstein, eds. Cost-effectiveness in health and medicine. New York: Oxford University Press, 1996:25-53.

5. Akehurst R. Making decisions on technology availability in the British National Health Service-why we need reliable models. Value in Health 2003;6:3-5.
6. Berki SE. In: Bashshur RL, Armstrong PA, Youssef ZI, eds. Telemedicine: explorations in the use of telecommunications in healthcare. Springfield, IL: Charles C. Thomas, 1975, and reproduced courtesy of Charles C Thomas, Ltd., Springfield, Illinois.

7. Gold MR, Siegel JE, Russell LB, Weinstein MC, eds. Cost-effectiveness in health and medicine. New York: Oxford Press, 1996.

8. Sculpher M, Pang F, Manca A, Drummond M, Golder S, Urdahl H, Davies L, Eastwood A. Generalisability in economic evaluation studies in healthcare: a review and case studies. Health Technology Assessment, NHS RED HTA Progamme 2004;8:124.

9. McKinsey Global Institute. US productivity growth 1995-2000: understanding the contribution of information technology relative to other factors. Washington DC: 2001.

10. Kennedy W, Laurier C, Malo J, Ghezzo H, L'Archevéque J, Contandriopoulos A. Does clinical trial subject selection restrict the ability to generalize use and cost of health services to "real life" subjects? Int I Tech Assess Health Care 2003;19:8-16. 
11. Ijzerman M, Reuzel R, Severens H. Pre-assessment to assess the match between results and decision makers' information needs. Int J Technol Assess Health Care 2003;19:17-27.

12. Alderson P, Green S, Higgins JPT, eds. Cochrane reviewers handbook 4.2.2. Chichester, UK: John Wiley \& Sons, Ltd., 2004.

13. Neumann PJ, Olchanski NV, Rosen AB, Greenberg D, Chapman R, Stone PW, Nadai J. Are published cost-utility analyses improving? Boston: Harvard School of Public Health, 2004.

14. Moja L, Telaro E, D’Amico R, Moschetti L, Coe L, Liberati A. Assessment of methodological quality of primary studies by systematic reviews: results of the metaquality cross sectional study. $\mathrm{Br} \mathrm{Med} \mathrm{I}$ 2005;300:1053-1057.

15. Hailey D, Roine R, Ohinmaa A. Systematic review of evidence for the benefits of telemedicine. L Telemed Telecare 2002;8(Suppl 1):1-30.

16. Roine R, Ohinmaa, A., Hailey, D. Assessing telemedicine: a systematic review of the literature. CMAJ, $\underline{\text { Can }}$ Med Assn I 2001;165:765-771.

17. Whitten PS, Mair FS, Haycox A, May CR, Williams TL, Hellmich S. Systematic review of cost effectiveness studies of telemedicine interventions. $\underline{\mathrm{Br} \mathrm{Med} \mathrm{I}}$ 2002;324:1434-1437.

18. Ohinmaa A, Hailey D, Roine R. The assessment of telemedicine-general principles and a systematic review: a health technology brief: economic evaluation methods. Alberta Heritage Foundation for Medical Research, 1999.

19. Hersh WR, Wallace JA, Patterson PK, et al. Telemedicine for the Medicare program. Oregon Health Sciences University AHRQ Publication No. 01-E012. Portland, OR: Oregon Health Sciences University, 2001.

20. Hersh WR, Wallace JA, Patterson PK, et al. Telemedicine for the Medicare population: pediatric, obstetric, and clinician-indirect home interventions. Oregon Health Sciences University AHRQ Publication No. 01-E060. Portland, OR: Oregon Health Sciences University, 2001.

21. Light R, Pillemer D. Summing up: the science of reviewing research. London: Harvard University Press, 1984.

22. Hakansson S, Gavelin, C. What do we really know about the cost-effectiveness of telemedicine? LTelemed Telecare 2000;6(Suppl 1):S133-S136.

23. Mair FS, Haycox A, May C, Williams T. A review of telemedicine cost-effectiveness studies. LTelemed Telecare 2000;6(Suppl 1):S38-S40.

24. Drummond MF. The contribution of health economics to cost-effective health care delivery. New York: Churchill Livingston, 1993.

25. Brantley D, Laney-Cummings K, Spivack R. Innovation, demand, and investment in telehealth. Washington DC: United States Department of Commerce, Office of Technology Policy, 2004.

26. McCue MJ, Mazmanian PE, Hampton CL, Marks TK, Fisher EJ, Parpart F, Malloy WN, Fisk KJ. Cost-minimization analysis: A follow-up study of a telemedicine program. Telemed I 1998;4:323-327.
27. Zincone LH Jr, Doty E, Balch DC. Financial analysis of telemedicine in a prison system. Telemed I 1997;3: 247-255.

28. Stoloff PH, Garcia FE, Thomason JE, Shia DS. A costeffectiveness analysis of shipboard telemedicine. Telemed I 1998;4:293-304.

29. Stoeger A, Strohmayr W, Giacomuzzi SM, Dessl A, Buchberger W, Jaschke W. A cost analysis of an emergency computerized tomography teleradiology system. I Telemed Telecare 1997;3:35-39.

30. Chodroff PH. A three-year review of telemedicine at the community level-clinical and fiscal results. I Telemed Telecare 1999;5(Suppl 1):S28-S30.

31. Doolittle G, Williams A, Harmon A, Allen A, Boysen CD, Wittman C, Mair F, Carlson E. A cost measurement study for a tele-oncology practice. LTelemed Telecare 1998;4:84-88.

32. Burgiss SG, Julius CE, Watson HW, Haynes BK, Buonocore E, Smith GT. Telemedicine for dermatology care in rural patients. Telemed I 1997;3:227-233.

33. Monnier J, Knapp RG, Frueh BC. Recent advances in telepsychiatry: an updated review. Psychiatr Serv 2003;54:1604-1609.

34. Grady BJ. A comparative cost analysis of an integrated military telemental health-care service. Telemed J e-Health 2002;8:293-300.

35. Hilty DM, Marks SL, Urness D, Yellowlees PM, Nesbitt TS. Clinical and educational telepsychiatry applications: a review. Can J Psychiatry 2004;49:12-23.

36. Grossman M. The demand for health: a theoretical and empirical investigation, New York: National Bureau of Economic Research, 1972.

37. Benatar D, Bondmass M, Ghitelman J, Avitall B. Outcomes of chronic heart failure. Arch Int Med 2003;163: 347-352.

38. Johnston B, Wheeler L, Deuser J, Sousa KH. Outcomes of the Kaiser Permanente tele-home health research project. Arch Fam Med 2000;9:40-45.

39. Dawson A, Cohen D, Candelier C, Jones G, Sanders J, Thompson A, Arnall C, Coles E. Domiciliary midwifery support in high-risk pregnancy incorporating telephonic fetal heart rate monitoring: a health technology randomized assessment. L Telemed Telecare 1999;5:220-230.

40. Loane MA, Bloomer SE, Corbett R, Eedy DJ, Evans C, Hicks N, Jacklin P, Lotery HE, Mathews C, Paisley J, Reid P, Steele K, Wootton R. A randomized controlled trial assessing the health economics of realtime teledermatology compared with conventional care: an urban versus rural perspective. L Telemed Telecare 2001;7:108-118.

41. Jacklin PB, Roberts JA, Wallace P, Haines A, Harrison R, Barber JA, Thompson SG, Lewis L, Currell R, Parker S, Wainwright P. Virtual outreach: economic evaluation of joint teleconsultations for patients referred by their general practitioner for a specialist opinion. Br Med I 2003;327:84.

42. Brown R, Hutton J, Nuijten M. Can unit costs be compared across Western European countries? Value in Health 2001;4:48. 
43. Drummond M, Barbieri M, Chancellor J, Willke D, Towse A, Jolain B. Are the results of economic evaluations generalizable? Evidence from studies of evidence from studies of pharmaceuticals in Western Europe. Presented at the 4th International Health Economics Association World Congress, 2003.

44. Sculpher M. Techniques to analyse variability across countries in multi-national economic evaluations. Presented at the 4th International Health Economics Association World Congress, 2003.

45. Thompson T, Brailer, D. The decade of health information technology: Delivering consumer-centric and information-rich health care. Washington, D.C.: Health and Human Services, 2004.

46. Bassoli F. Justifying where the dollars go. Health Data Manag Feb. 1998.

47. Capital Planning and IT Investment Committee. ROI and the value puzzle. Washington, D.C.: Federal CIO Council, 1999.

48. Glick H. Statistical methods in economic evaluation. Philadelphia: University of Pennsylvania, 2003.

49. Strosberg MA, Wiener JM, Baker R, Fein IA, eds. Rationing America's medical care: The Oregon plan and beyond. Washington DC: The Brookings Institution, 1992.

50. Torrence GW, Siegel JE, Luce BR. In: Gold MR, Siegel JE, Russell LB, Weinstein MC, eds. Cost-effectiveness in health and medicine. New York: Oxford University Press, 1996:54-81.
51. United States Government Accounting Office. Department. Executive guide-measuring performance and demonstrating results of information technology investments. Washington, D.C.: 1998.

52. Snodgrass NB, Jones PC, Meyer S, Roos DC, Safran C. Functional economic analysis: a technique for identifying technology investment opportunities. Presented at the The Health Information Management Systems Society (HIMSS), 1997.

53. Casson M. Information and organization: a new perspective on theory of the firm. New York: Oxford University Press, 1997.

54. Simon H. The sciences of the artificial. Cambridge, MA: MIT Press, 1969.

55. United States Department of Defense. Functional economic analysis (FEA) guidebook. Electronic College of Process Innovation. http://www.dtic.mil, 2003.

56. Pearce DW, ed. The MIT dictionary of modern economics. 3rd ed. Cambridge: The MIT Press, 1986.

Address reprint requests to: Tim Reardon, Ph.D.

TG Reardon \& Associates, LLC 1232 Prospect Avenue Ann Arbor, MI 48104

E-mail: treardon@umich.edu 


\section{This article has been cited by:}

1. Michael Ries. 2011. Tele-ICU: A New Paradigm in Critical Care. International Anesthesiology Clinics 47:1, 153-170. [CrossRef]

2. Micaela Brown , Nicola Shaw . 2008. Evaluation Practices of a Major Canadian Telehealth Provider: Lessons and Future Directions for the FieldEvaluation Practices of a Major Canadian Telehealth Provider: Lessons and Future Directions for the Field. Telemedicine and e-Health 14:8, 769-774. [Abstract] [PDF] [PDF Plus]

3. Kimmo Murto, Gregory L Bryson, Ibrahim Abushahwan, Jim King, David Moher, Khaled El-Emam, William Splinter. 2008. Parents are reluctant to use technological means of communication in pediatric day care. Canadian Journal of Anesthesia/Journal canadien d'anesthésie 55:4, 214-222. [CrossRef]

4. Mengistu Kifle, Victor W. A. Mbarika, Pratim Datta. 2006. Interplay of cost and adoption of tele-medicine in Sub-Saharan Africa: The case of tele-cardiology in Ethiopia. Information Systems Frontiers 8:3, 211-223. [CrossRef]

5. Eckart Fleck, Claus W Biermann, Uta Bruns. 2006. FOREWORD. Disease Management \& Health Outcomes 14:Suppl 1, vi???viii. [CrossRef]

6. Penny Jennett, Mamoru Watanabe. 2006. Healthcare and Telemedicine: Ongoing and Evolving Challenges. Disease Management \& Health Outcomes 14:Suppl 1, 9???13. [CrossRef] 